\title{
Delayed Diagnosis of Tuberculosis Mistaken for Tinea Corporis in a Healthy Adult
}

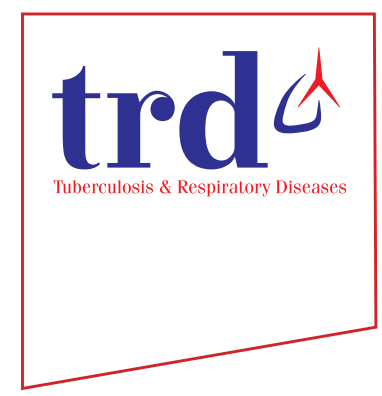

\author{
Jae-Wang Kim, M.D., Ph.D. ${ }^{1}{ }^{(i)}$, Jeong Rae Yoo, M.D. ${ }^{2}$, Hyunjoo Oh, M.D. ${ }^{2}$, Misun Kim, M.D. ${ }^{2}$ and \\ Sang Taek Heo, M.D., Ph.D. ${ }^{2}$ (i) \\ Departments of ${ }^{1}$ Dermatology and ${ }^{2}$ Internal Medicine, Jeju National University College of Medicine, Jeju, Republic of Korea
}

Mycobacterium tuberculosis infection is a serious health

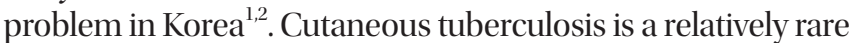
manifestation of tuberculosis infection, comprising $<2 \%$ of all extrapulmonary tuberculosis cases ${ }^{3,4}$. In recent years, this infection has become rare, and frequent misdiagnosis leads to delayed diagnosis ${ }^{5}$. General physicians are more familiar with cutaneous fungal diseases such as tinea corporis than with tuberculosis verrucosa cutis. Therefore, clinicians should be aware of this disease for timely diagnosis. We have reported a case of concurrent tuberculosis in the lung and on the skin of the buttock.

A 41-year-old man presented to our clinic with a 4-year history of itching and mild tenderness in the perianal area. He was previously diagnosed with tinea corporis at a dermatol- ogy clinic and had received topical antifungal treatment for four years. On presentation, he denied experiencing fever, chills, non-productive and productive cough, weight loss, and night sweats. Physical examination revealed elevated coalescent erythematous scaling plaques $(8 \mathrm{~cm} \times 8 \mathrm{~cm})$ on the right buttock (Figure 1A). Laboratory examination revealed human immunodeficiency virus negativity. Biopsy of the lesion revealed pseudoepitheliomatous hyperplasia with granulomatous inflammation in the superficial dermis (Figure 1B); periodic acid-Schiff and acid-fast bacilli stains were negative. Chest radiography revealed focal haziness in the right upper lobe, and chest computed tomography showed nodules and peribronchial infiltration in the right upper lobe (Figure 2). M. tuberculosis was isolated from the cutaneous tissue and
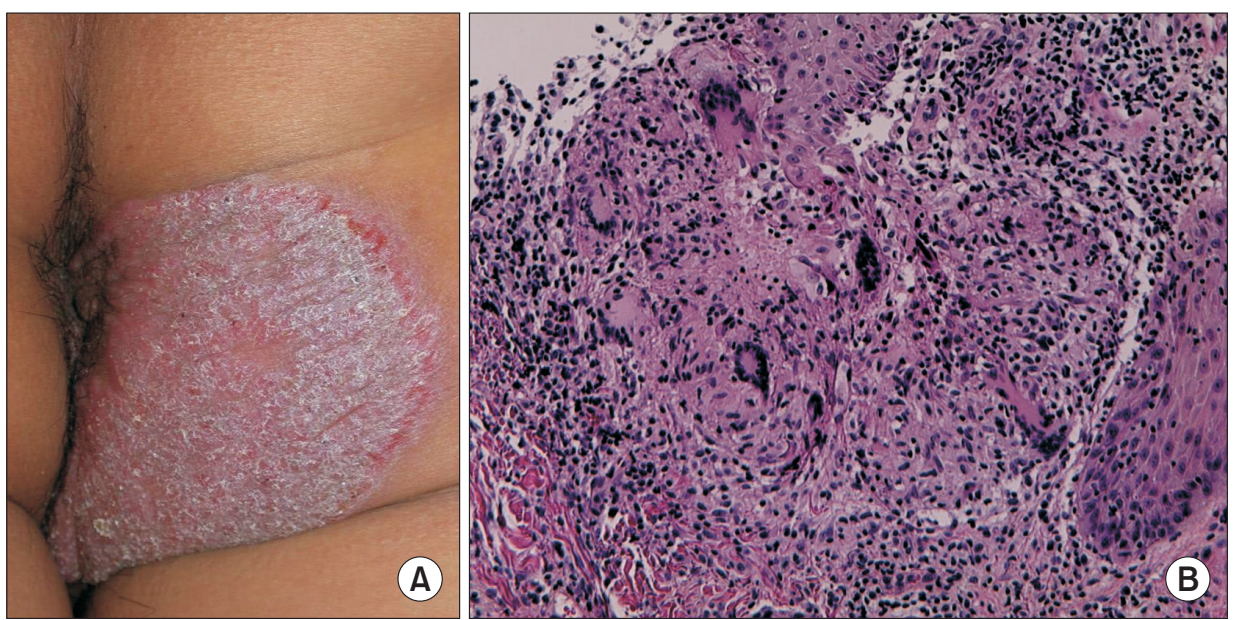

Figure 1. (A) Elevated coalescent erythematous scaling plaques on the right buttock. (B) Histopathological findings showing pseudoepitheliomatous hyperplasia with granulomatous inflammation in the superficial dermis (H\&E stain, $\times 100)$. The results of the acid-fast bacilli strain and polymerase chain reaction for M. tuberculosis were negative.

Address for correspondence: Sang Taek Heo, M.D., Ph.D.

Department of Internal Medicine, Jeju National University College of Medicine, 102 Jejudaehang-ro, Jeju 54987, Republic of Korea Phone: 82-64-717-1296, Fax: 82-64-717-1131, E-mail: neosangtaek@naver.com

Received: Mar. 12, 2021, Revised: Apr. 2, 2021, Accepted: Apr. 14, 2021, Published online: Apr. 15, 2021

(c) It is identical to the Creative Commons Attribution Non-Commercial License (http://creativecommons.org/licenses/by-nc/4.0/).

The Korean Academy of Tuberculosis and Respiratory Diseases. 

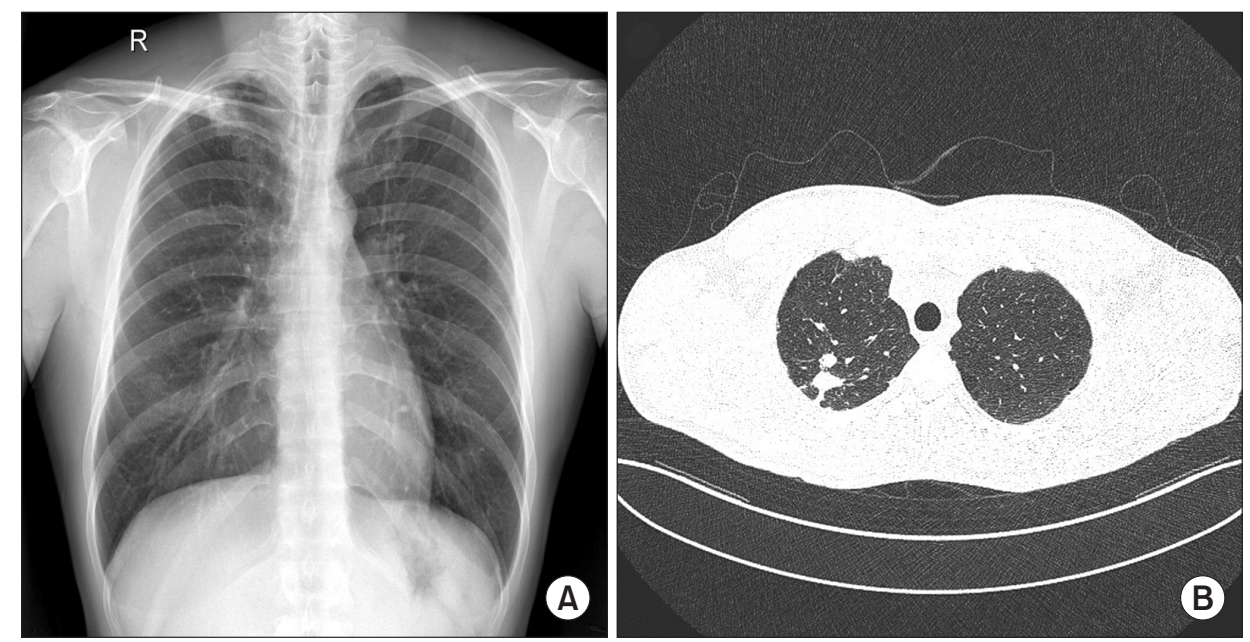

sputum; it was not resistant to anti-tuberculosis agents. Thus, treatment with anti-tuberculosis agents such as isoniazid, rifampicin, ethambutol, and pyrazinamide was initiated for 2 months, which was followed by isoniazid, and rifampicin therapy for 4 months. Subsequently, the lesion completely resolved without recurrence.

\section{Authors' Contributions}

Conceptualization: Kim JW, Heo ST, Yoo JR. Methodology: Yoo JR, Kim JW. Formal analysis: Kim M, Oh H. Data curation: Kim M, Oh H. Software: Yoo JR. Validation: Heo ST, Kim JW. Investigation: Kim M, Oh H. Writing - original draft preparation: Kim JW, Heo ST. Writing - review and editing: Kim JW, Heo ST. Approval of final manuscript: all authors.

\section{Conflicts of Interest}

No potential conflict of interest relevant to this article was reported.
Figure 2. (A) Chest radiography showing focal consolidation in the right upper lobe. (B) Chest computed tomography showing a few nodular lesions and peribronchial infiltrates in the right upper lobe.

\section{Funding}

This work was supported by the 2021 education, research and student guidance grant funded by Jeju National University.

\section{References}

1. Cho KS. Tuberculosis control in the Republic of Korea. Epidemiol Health 2018;40:e2018036.

2. Kwon OY. Public health center on tuberculosis management in Korea: from 1945 to the late 1970s. Uisahak 2019;28:72154.

3. van Zyl L, du Plessis J, Viljoen J. Cutaneous tuberculosis overview and current treatment regimens. Tuberculosis (Edinb) 2015;95:629-38.

4. Santos JB, Figueiredo AR, Ferraz CE, Oliveira MH, Silva PG, Medeiros VL. Cutaneous tuberculosis: epidemiologic, etiopathogenic and clinical aspects - part I. An Bras Dermatol 2014;89:219-28.

5. Chen Q, Chen W, Hao F. Cutaneous tuberculosis: a great imitator. Clin Dermatol 2019;37:192-9. 